Acta Crystallographica Section E

Structure Reports

Online

ISSN 1600-5368

\section{Marten G. Barker,† M. Grazia Francesconi and Claire Wilson*}

School of Chemistry, University of Nottingham, Nottingham NG7 2RD, England

+ Deceased.

Correspondence e-mail: claire.wilson@nott.ac.uk

\section{Key indicators}

Single-crystal X-ray study

$T=150 \mathrm{~K}$

Mean $\sigma(\mathrm{O}-\mathrm{Ba})=0.008 \AA$

$R$ factor $=0.022$

$w R$ factor $=0.051$

Data-to-parameter ratio $=31.3$

For details of how these key indicators were automatically derived from the article, see http://journals.iucr.org/e.

\title{
Barium oxide iodide
}

Barium oxide iodide, $\mathrm{Ba}_{4} \mathrm{OI}_{6}$, has been prepared by a solidstate reaction and shown to be isostructural with both $A_{4} \mathrm{OCl}_{6}$, where $A$ is $\mathrm{Ba}$ or $\mathrm{Sr}$, and $\mathrm{Sr}_{4} \mathrm{OI}_{6}$.

\section{Comment}

Alkaline earth oxide chlorides and oxide bromides, of general formula $A_{4} \mathrm{O} X_{6}\left(A=\right.$ alkaline earth; $\left.X=\mathrm{Cl}^{-}, \mathrm{Br}^{-}\right)$are known for their luminescence properties, when the alkaline earth site is doped with small amounts of $\mathrm{Eu}^{2+}$ or $\mathrm{Pb}^{2+}$ (Schipper et al., 1992). We have succeeded in extending this family to the barium oxide iodide compound $\mathrm{Ba}_{4} \mathrm{OI}_{6}$. $\mathrm{Ba}_{4} \mathrm{OI}_{6}$ was prepared by a solid-state reaction and is isostructural with $\mathrm{Sr}_{4} \mathrm{OCl}_{6}$ (Hagemann et al., 1996), $\mathrm{Ba}_{4} \mathrm{OCl}_{6}$ (Bergerhoff \& Goost, 1970) and $\mathrm{Sr}_{4} \mathrm{OI}_{6}$ (Barker et al., 2001). The $\mathrm{O}$ atom is four-coordinated by Ba cations (see Fig. 1), the iodine is four- and fivecoordinated by Ba cations (Figs. 2 and 3) and the $\mathrm{Ba}$ is eightcoordinated by one oxygen and seven iodine anions at one site, and seven-coordinated by one oxygen and six iodine anions at the other (Figs. 4 and 5). The overall structure is shown in Fig. 1 of Barker et al. (2001).

\section{Experimental}

$\mathrm{BaO}$ and $\mathrm{BaI}_{2}$ powders were mixed in stoichiometric proportions and placed in a nickel crucible. The mixture was then heated at $1273 \mathrm{~K}$ for $24 \mathrm{~h}$ in a silica tube, under flowing nitrogen. The product was cooled to room temperature at a rate of $1 \mathrm{~K} \mathrm{~h}^{-1}$.

\section{Crystal data}

$\mathrm{Ba}_{4} \mathrm{OI}_{6}$

$M_{r}=1326.72$

Hexagonal, $P 6_{3} m c$

$a=10.838$ (4) §

$c=8.410(3) \AA$

$V=855.5(9) \AA^{3}$

$Z=2$

$D_{x}=5.150 \mathrm{Mg} \mathrm{m}^{-3}$

Data collection

Bruker SMART1000 CCD areadetector diffractometer

$\omega$ scans

Absorption correction: multi-scan (SADABS; Bruker, 1996)

$T_{\min }=0.077, T_{\max }=0.137$ 4900 measured reflections

842 independent reflections

\section{Refinement}

Refinement on $F^{2}$

$R\left[F^{2}>2 \sigma\left(F^{2}\right)\right]=0.022$

$w R\left(F^{2}\right)=0.051$

$S=1.09$

782 reflections

25 parameters
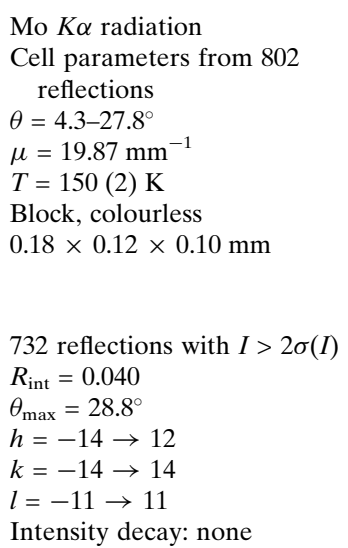

$w=1 /\left[\sigma^{2}\left(F_{o}^{2}\right)+(0.0263 P)^{2}\right]$

where $P=\left(F_{o}^{2}+2 F_{c}^{2}\right) / 3$

$(\Delta / \sigma)_{\max }=0.001$

$\Delta \rho_{\max }=0.82{\mathrm{e} \AA^{-3}}^{-3}$

$\Delta \rho_{\min }=-1.20{\mathrm{e} \AA^{-3}}^{-3}$

Absolute structure: Flack (1983)

Flack parameter $=-0.06(10)$
Received 13 February 2001 Accepted 20 April 2001 Online 22 May 2001
(C) 2001 International Union of Crystallography Printed in Great Britain - all rights reserved 


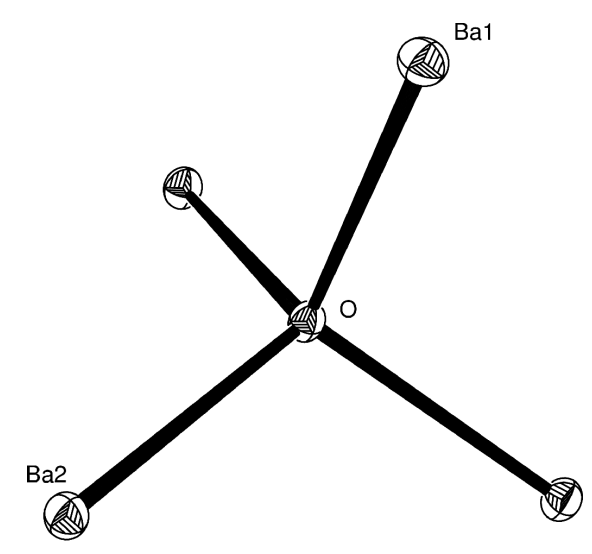

Figure 1

A view showing the tetrahedral coordination around the $\mathrm{O}$ atom. Displacement ellipsoids are drawn at the $50 \%$ probability level.

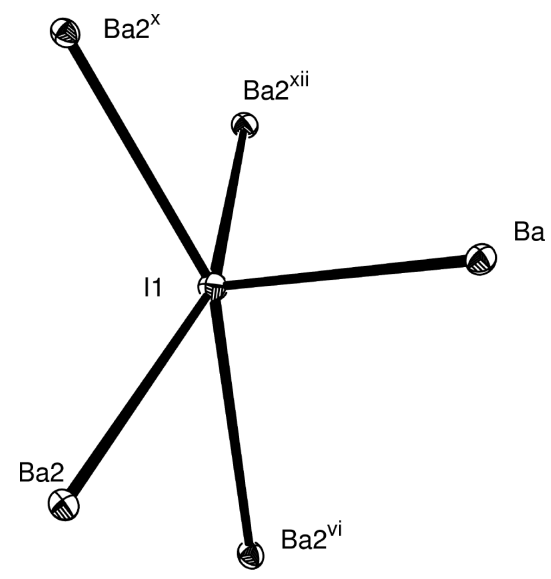

Figure 2

A view showing coordination around atom I1. Displacement ellipsoids are drawn at the $50 \%$ probability level. [Symmetry codes: (vi) $1-y$, $1+x-y, z ;(\mathrm{x}) y,-x+y, \frac{1}{2}+z ;(\mathrm{xii}) 1-x, 1-y, \frac{1}{2}+z$.]

\section{Table 1}

Selected geometric parameters $\left(\AA{ }^{\circ}\right)$.

\begin{tabular}{llll}
\hline $\mathrm{Ba} 1-\mathrm{O}^{\mathrm{i}}$ & $2.542(12)$ & $\mathrm{Ba} 2-\mathrm{I}^{\mathrm{iv}}$ & $3.5115(16)$ \\
$\mathrm{Ba} 1-\mathrm{I} 1^{\mathrm{ii}}$ & $3.5848(17)$ & $\mathrm{Ba} 2-\mathrm{I}^{\mathrm{v}}$ & $3.5560(15)$ \\
$\mathrm{Ba} 1-\mathrm{I} 2$ & $3.6927(18)$ & $\mathrm{Ba} 2-\mathrm{I} 1$ & $3.6781(15)$ \\
$\mathrm{Ba} 2-\mathrm{O}$ & $2.555(4)$ & $\mathrm{O}-\mathrm{Ba} 1^{\mathrm{iii}}$ & $2.542(12)$ \\
$\mathrm{Ba} 2-\mathrm{I} 2^{\mathrm{iii}}$ & $3.467(2)$ & & \\
\hline
\end{tabular}

Symmetry codes: (i) $x, y, z-1$; (ii) $y, 1-x+y, z-\frac{1}{2}$; (iii) $x, y, 1+z$; (iv) $y,-x+y, \frac{1}{2}+z ;(\mathrm{v}) 1-x, 1-y, z-\frac{1}{2}$.

The origin was fixed by application of a floating origin restraint which effectively fixes the centre of gravity of the structure in the polar-axis direction. This leads to smaller correlations than fixing a single atom in structures with no dominant heavy atom (Flack \& Schwarzenbach, 1988).

Data collection: SMART Bruker, 1998); cell refinement: SAINT (Bruker, 2000); data reduction: SAINT and SHELXL97 (Sheldrick, 1997); program(s) used to solve structure: SIR97 (Altomare et al., 1999); program(s) used to refine structure: SHELXL97 and WINGX (Farrugia, 1999); molecular graphics: ORTEP-3 (Farrugia, 1997);

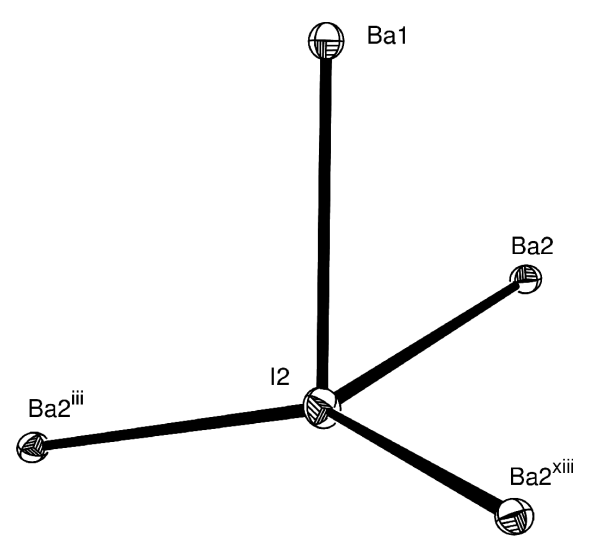

Figure 3

A view showing the coordination around atom I2. Displacement ellipsoids are drawn at the $50 \%$ probability level. [Symmetry codes: (iii) $x-y, x, z-\frac{1}{2}$; (xiii) $y,-x+y, z-\frac{1}{2}$.]

Figure 4

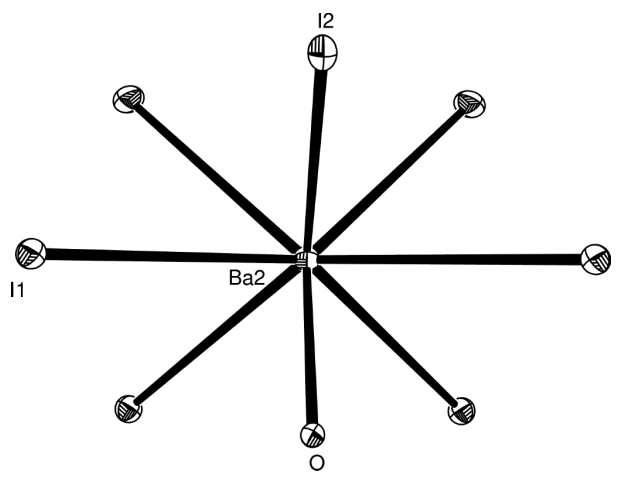

A view showing the coordination around atom Ba2. Displacement ellipsoids are drawn at the $50 \%$ probability level.

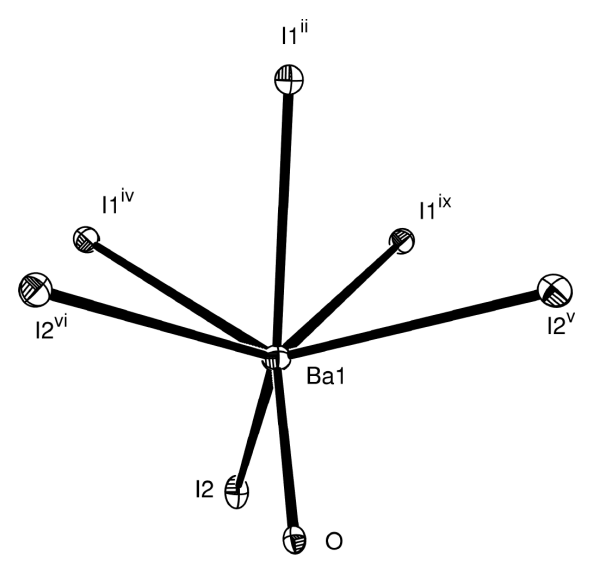

Figure 5

A view showing the coordination around atom Ba1. Displacement ellipsoids are drawn at the $50 \%$ probability level. [Symmetry codes: (ii) $y$, $1-x+y, z-\frac{1}{2}$; (iv) $1-x, 1-y, z-\frac{1}{2}$; (v) $-x+y, 1-x$, $z$; (vi) $1-y$, $1+x-y, z ;$ (ix) $x-y, x, \frac{1}{2}+z$.]

software used to prepare material for publication: SHELXL97 and PLATON (Spek, 2001).

We wish to thank the EPSRC for support. 


\section{inorganic papers}

\section{References}

Altomare, A., Burla, M. C., Camalli, M., Cascarano, G, Giacovazzo, C., Guagliardi, A., Moliterni, A. G. G., Polidori, G. \& Spagna, R. (1999). J. Appl. Cryst. 32, 115-119.

Barker, M. G., Francesconi, M. G., Shutt, T. H. \& Wilson C. (2001). Acta Cryst. E57, i44-45.

Bergerhoff, G. \& Goost, L. (1970). Acta Cryst. B26, 19-23.

Bruker (1996). SADABS. Bruker AXS Inc., Madison, Wisconsin, USA.

Bruker (1998). SMART Area-Detector Software Package. Version 5.054. Bruker AXS Inc., Madison, Wisconsin, USA.
Bruker (2000). SAINT Frame Integration Software. Version 6.02a. Bruker AXS Inc., Madison, Wisconsin, USA.

Farrugia, L. J. (1997). J. Appl. Cryst. 30, 565.

Farrugia, L. J. (1999). J. Appl. Cryst. 32, 837-838.

Flack, H. D. (1983). Acta Cryst. A39, 876-881.

Flack, H. D \& Schwarzenbach, D. (1988). Acta Cryst. A44, 499-506.

Hagemann, H., Kubel, F. \& Bill, H. (1996). Eur. J. Solid State Inorg. Chem. 33 1101-1109.

Schipper, W. J., Vroon, Z. A. E. P., Blasse, G. \& Schleid, T. (1992). Chem. Mater. 4, 688-692.

Sheldrick, G. M.(1997). SHELXL97. University of Göttingen, Germany.

Spek, A. L. (2001). PLATON. University of Utrecht, The Netherlands. 


\section{supporting information}

Acta Cryst. (2001). E57, i41-i43 [https://doi.org/10.1107/S1600536801006614]

Barium oxide iodide

Marten G. Barker, M. Grazia Francesconi and Claire Wilson

(I)

\section{Crystal data}

$\mathrm{Ba}_{4} \mathrm{OI}_{6}$

$M_{r}=1326.72$

Hexagonal, $P 6_{3} m c$

$a=10.838(4) \AA$

$c=8.410(3) \AA$

$V=855.5(9) \AA^{3}$

$Z=2$

$F(000)=1100$

$D_{\mathrm{x}}=5.150 \mathrm{Mg} \mathrm{m}^{-3}$

Mo $K \alpha$ radiation, $\lambda=0.71069 \AA$

Cell parameters from 802 reflections

$\theta=4.3-27.8^{\circ}$

$\mu=19.87 \mathrm{~mm}^{-1}$

$T=150 \mathrm{~K}$

Block, colourless

$0.18 \times 0.12 \times 0.10 \mathrm{~mm}$

Data collection

Bruker SMART1000 CCD area-detector diffractometer

Radiation source: fine-focus sealed tube Graphite monochromator

$\omega$ scans

Absorption correction: multi-scan

(SADABS; Bruker, 1996)

$T_{\min }=0.077, T_{\max }=0.137$

4900 measured reflections

842 independent reflections

732 reflections with $I>2 \sigma(I)$

$R_{\text {int }}=0.040$

$\theta_{\text {max }}=28.8^{\circ}, \theta_{\text {min }}=2.2^{\circ}$

$h=-14 \rightarrow 12$

$k=-14 \rightarrow 14$

$l=-11 \rightarrow 11$

\section{Refinement}

Refinement on $F^{2}$

Least-squares matrix: full

$R\left[F^{2}>2 \sigma\left(F^{2}\right)\right]=0.022$

$w R\left(F^{2}\right)=0.051$

$S=1.09$

782 reflections

25 parameters

1 restraint

Primary atom site location: structure-invariant direct methods

Secondary atom site location: difference Fourier

$$
\begin{gathered}
\text { map } \\
w=1 /\left[\sigma^{2}\left(F_{\mathrm{o}}{ }^{2}\right)+(0.0263 P)^{2}\right] \\
\text { where } \left.P=\left(F_{\mathrm{o}}{ }^{2}+2 F_{\mathrm{c}}\right)^{2}\right) / 3 \\
(\Delta / \sigma)_{\max }=0.001 \\
\Delta \rho_{\max }=0.82 \mathrm{e} \AA^{-3} \\
\Delta \rho_{\min }=-1.20 \mathrm{e} \AA^{-3}
\end{gathered}
$$

Absolute structure: Flack (1983)

Absolute structure parameter: -0.06 (10)

Special details

Geometry. All e.s.d.'s (except the e.s.d. in the dihedral angle between two l.s. planes) are estimated using the full covariance matrix. The cell e.s.d.'s are taken into account individually in the estimation of e.s.d.'s in distances, angles and torsion angles; correlations between e.s.d.'s in cell parameters are only used when they are defined by crystal symmetry. An approximate (isotropic) treatment of cell e.s.d.'s is used for estimating e.s.d.'s involving 1.s. planes. 
Refinement. Refinement of $F^{2}$ against ALL reflections. The weighted $R$-factor $w R$ and goodness of fit $S$ are based on $F^{2}$, conventional $R$-factors $R$ are based on $F$, with $F$ set to zero for negative $F^{2}$. The threshold expression of $F^{2}>\sigma\left(F^{2}\right)$ is used only for calculating $R$-factors(gt) etc. and is not relevant to the choice of reflections for refinement. $R$-factors based on $F^{2}$ are statistically about twice as large as those based on $F$, and $R$ - factors based on ALL data will be even larger.

Fractional atomic coordinates and isotropic or equivalent isotropic displacement parameters $\left(\AA^{2}\right)$

\begin{tabular}{lllll}
\hline & $x$ & $y$ & $z$ & $U_{\text {iso }} * / U_{\text {eq }}$ \\
\hline Ba1 & 0.3333 & 0.6667 & $0.02342(13)$ & $0.0133(2)$ \\
Ba2 & $0.20359(3)$ & $0.40719(6)$ & $0.62927(8)$ & $0.01196(14)$ \\
I1 & $0.53228(3)$ & $0.46772(3)$ & $0.82627(8)$ & $0.01280(15)$ \\
I2 & $0.13667(3)$ & $0.27334(6)$ & $0.01355(8)$ & $0.01651(16)$ \\
O & 0.3333 & 0.6667 & $0.7212(15)$ & $0.010(2)$ \\
\hline
\end{tabular}

Atomic displacement parameters $\left(\AA^{2}\right)$

\begin{tabular}{lllllll}
\hline & $U^{11}$ & $U^{22}$ & $U^{33}$ & $U^{12}$ & $U^{13}$ & $U^{23}$ \\
\hline Ba1 & $0.0142(3)$ & $0.0142(3)$ & $0.0113(5)$ & $0.00711(15)$ & 0.000 & 0.000 \\
Ba2 & $0.0109(2)$ & $0.0100(3)$ & $0.0147(3)$ & $0.00499(14)$ & $-0.00039(13)$ & $-0.0008(3)$ \\
$\mathrm{I} 1$ & $0.0127(2)$ & $0.0127(2)$ & $0.0130(3)$ & $0.0064(3)$ & $-0.00047(14)$ & $0.00047(14)$ \\
$\mathrm{I} 2$ & $0.0126(2)$ & $0.0184(3)$ & $0.0204(3)$ & $0.00919(17)$ & $0.00145(15)$ & $0.0029(3)$ \\
$\mathrm{O}$ & $0.008(3)$ & $0.008(3)$ & $0.013(6)$ & $0.0041(16)$ & 0.000 & 0.000 \\
\hline
\end{tabular}

Geometric parameters $\left(\AA,{ }^{\circ}\right)$

\begin{tabular}{|c|c|c|c|}
\hline $\mathrm{Ba} 1-\mathrm{O}^{\mathrm{i}}$ & $2.542(12)$ & $\mathrm{Ba} 2-\mathrm{I}^{\mathrm{v}}$ & $3.6781(15)$ \\
\hline $\mathrm{Ba} 1-\mathrm{I} 1^{\mathrm{ii}}$ & $3.5848(17)$ & $\mathrm{Ba} 2-\mathrm{I} 1$ & $3.6781(15)$ \\
\hline $\mathrm{Ba} 1-\mathrm{I} 1^{\mathrm{iii}}$ & $3.5848(17)$ & $\mathrm{Ba} 2-\mathrm{Ba} 1^{\mathrm{ix}}$ & $4.113(2)$ \\
\hline $\mathrm{Ba} 1-\mathrm{I} 1^{\mathrm{iv}}$ & $3.5848(17)$ & $\mathrm{Ba} 2-\mathrm{Ba}^{\mathrm{vi}}$ & $4.218(2)$ \\
\hline $\mathrm{Ba} 1-\mathrm{I} 2^{\mathrm{v}}$ & $3.6927(18)$ & $\mathrm{Ba} 2-\mathrm{Ba}^{\mathrm{v}}$ & $4.218(2)$ \\
\hline $\mathrm{Ba} 1-\mathrm{I} 2^{\mathrm{vi}}$ & $3.6927(18)$ & $\mathrm{I} 1-\mathrm{Ba} 2^{\mathrm{x}}$ & $3.5560(15)$ \\
\hline $\mathrm{Ba} 1-\mathrm{I} 2$ & $3.6927(18)$ & $\mathrm{I} 1-\mathrm{Ba} 2^{\mathrm{xii}}$ & $3.5560(15)$ \\
\hline $\mathrm{Ba} 1-\mathrm{Ba}^{\mathrm{vii}}$ & $4.113(2)$ & $\mathrm{I} 1-\mathrm{Ba} 1^{\mathrm{xii}}$ & $3.5848(17)$ \\
\hline $\mathrm{Ba} 1-\mathrm{Ba} 2^{\mathrm{i}}$ & $4.113(2)$ & $\mathrm{I} 1-\mathrm{Ba} 2^{\mathrm{vi}}$ & $3.6781(15)$ \\
\hline $\mathrm{Ba} 1-\mathrm{Ba} 2^{\text {viii }}$ & $4.113(2)$ & $\mathrm{I} 2-\mathrm{Ba} 2^{\mathrm{i}}$ & $3.467(2)$ \\
\hline $\mathrm{Ba} 2-\mathrm{O}$ & $2.555(4)$ & $\mathrm{I} 2-\mathrm{Ba} 2^{\mathrm{iii}}$ & $3.5115(16)$ \\
\hline $\mathrm{Ba} 2-\mathrm{I} 2^{\mathrm{ix}}$ & $3.467(2)$ & $\mathrm{I} 2-\mathrm{Ba} 2^{\mathrm{xiii}}$ & $3.5115(16)$ \\
\hline $\mathrm{Ba} 2-\mathrm{I} 2^{\mathrm{x}}$ & $3.5115(16)$ & $\mathrm{O}-\mathrm{Ba} 1^{\mathrm{ix}}$ & $2.542(12)$ \\
\hline $\mathrm{Ba} 2-\mathrm{I} 2^{\mathrm{xi}}$ & $3.5115(16)$ & $\mathrm{O}-\mathrm{Ba} 2^{\mathrm{v}}$ & $2.555(4)$ \\
\hline $\mathrm{Ba} 2-\mathrm{I}^{\mathrm{iii}}$ & $3.5560(15)$ & $\mathrm{O}-\mathrm{Ba} 2^{\mathrm{vi}}$ & $2.555(4)$ \\
\hline $\mathrm{Ba} 2-\mathrm{I}^{\mathrm{iv}}$ & $3.5560(15)$ & & \\
\hline $\mathrm{O}^{\mathrm{i}}-\mathrm{Ba} 1-\mathrm{I} 1^{\mathrm{ii}}$ & $135.27(3)$ & $\mathrm{I} 2^{\mathrm{ix}}-\mathrm{Ba} 2-\mathrm{I} 1^{\mathrm{v}}$ & $68.66(2)$ \\
\hline $\mathrm{O}-\mathrm{Ba} 1-\mathrm{I} 1^{\mathrm{iii}}$ & $135.27(3)$ & $\mathrm{I} 2^{\mathrm{x}}-\mathrm{Ba} 2-\mathrm{I} 1^{\mathrm{v}}$ & 142.459 (19) \\
\hline $\mathrm{I} 1^{\mathrm{ii}}-\mathrm{Ba} 1-\mathrm{I} 1^{\mathrm{iii}}$ & $75.10(4)$ & $\mathrm{I} 2^{\mathrm{xi}}-\mathrm{Ba} 2-\mathrm{I} 1^{\mathrm{v}}$ & $71.35(2)$ \\
\hline $\mathrm{O}^{\mathrm{i}}-\mathrm{Ba} 1-\mathrm{I} 1^{\mathrm{iv}}$ & $135.27(3)$ & 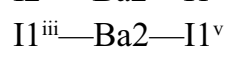 & $74.43(4)$ \\
\hline 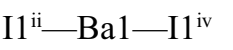 & $75.10(4)$ & $\mathrm{I} 1^{\mathrm{iv}}-\mathrm{Ba} 2-\mathrm{I} 1^{\mathrm{v}}$ & 144.294 (19) \\
\hline 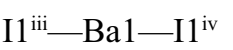 & $75.10(4)$ & $\mathrm{O}-\mathrm{Ba} 2-\mathrm{I} 1$ & $73.53(11)$ \\
\hline
\end{tabular}




\begin{tabular}{|c|c|c|c|}
\hline $\mathrm{O}^{\mathrm{i}}-\mathrm{Ba} 1-\mathrm{I} 2^{\mathrm{v}}$ & $88.71(2)$ & $\mathrm{I} 2^{\mathrm{ix}}-\mathrm{Ba} 2-\mathrm{I} 1$ & $68.66(2)$ \\
\hline 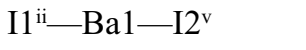 & $70.379(15)$ & $\mathrm{I} 2^{\mathrm{x}}-\mathrm{Ba} 2-\mathrm{I} 1$ & $71.35(2)$ \\
\hline $\mathrm{I} 1^{\mathrm{iii}}-\mathrm{Ba} 1-\mathrm{I} 2^{\mathrm{v}}$ & $70.379(16)$ & $\mathrm{I} 2^{\mathrm{xi}}-\mathrm{Ba} 2-\mathrm{I} 1$ & $142.459(19)$ \\
\hline $\mathrm{I}^{1 \mathrm{iv}}-\mathrm{Ba} 1-\mathrm{I} 2^{\mathrm{v}}$ & $136.01(4)$ & 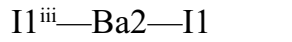 & $144.29(2)$ \\
\hline $\mathrm{O}-\mathrm{Ba} 1-\mathrm{I} 2^{\mathrm{vi}}$ & $88.71(2)$ & $\mathrm{I} 1{ }^{\mathrm{iv}}-\mathrm{Ba} 2-\mathrm{I} 1$ & $74.43(4)$ \\
\hline $\mathrm{I} 1^{\mathrm{ii}}-\mathrm{Ba} 1-\mathrm{I} 2^{\mathrm{vi}}$ & $70.379(16)$ & $\mathrm{I} 1^{\mathrm{v}}-\mathrm{Ba} 2-\mathrm{I} 1$ & $123.13(4)$ \\
\hline $\mathrm{I} 1^{\mathrm{iii}}-\mathrm{Ba} 1-\mathrm{I} 2^{\mathrm{vi}}$ & $136.01(4)$ & $\mathrm{O}-\mathrm{Ba} 2-\mathrm{Ba} 1^{\mathrm{ix}}$ & $36.1(3)$ \\
\hline $\mathrm{I} 1^{\mathrm{iv}}-\mathrm{Ba} 1-\mathrm{I} 2^{\mathrm{vi}}$ & $70.379(16)$ & $\mathrm{I} 2^{\mathrm{ix}}-\mathrm{Ba} 2-\mathrm{Ba} 1^{\mathrm{ix}}$ & $57.55(4)$ \\
\hline $\mathrm{I} 2^{\mathrm{v}}-\mathrm{Ba} 1-\mathrm{I} 2^{\mathrm{vi}}$ & $119.950(2)$ & $\mathrm{I} 2^{\mathrm{x}}-\mathrm{Ba} 2-\mathrm{Ba} 1^{\mathrm{ix}}$ & $130.653(17)$ \\
\hline $\mathrm{O}^{\mathrm{i}-\mathrm{Ba} 1-\mathrm{I} 2}$ & $88.71(2)$ & $\mathrm{I} 2^{\mathrm{xi}}-\mathrm{Ba} 2-\mathrm{Ba} 1^{\mathrm{ix}}$ & $130.653(17)$ \\
\hline $\mathrm{I} 1^{\mathrm{ii}}-\mathrm{Ba} 1-\mathrm{I} 2$ & $136.01(4)$ & 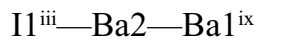 & $112.46(4)$ \\
\hline $\mathrm{I} 1{ }^{\mathrm{iii}}-\mathrm{Ba} 1-\mathrm{I} 2$ & $70.379(16)$ & $\mathrm{I} 1^{\mathrm{iv}}-\mathrm{Ba} 2-\mathrm{Ba} 1^{\mathrm{ix}}$ & $112.46(4)$ \\
\hline $\mathrm{I} 1^{\mathrm{iv}}-\mathrm{Ba} 1-\mathrm{I} 2$ & $70.379(16)$ & $\mathrm{I} 1^{\mathrm{v}}-\mathrm{Ba} 2-\mathrm{Ba} 1^{\mathrm{ix}}$ & $62.97(2)$ \\
\hline $\mathrm{I} 2^{\mathrm{v}}-\mathrm{Ba} 1-\mathrm{I} 2$ & $119.950(2)$ & $\mathrm{I} 1-\mathrm{Ba} 2-\mathrm{Ba} 1^{\mathrm{ix}}$ & $62.97(2)$ \\
\hline $\mathrm{I} 2^{\mathrm{vi}}-\mathrm{Ba} 1-\mathrm{I} 2$ & $119.950(2)$ & $\mathrm{O}-\mathrm{Ba} 2-\mathrm{Ba}^{\mathrm{vi}}$ & $34.36(12)$ \\
\hline $\mathrm{O}^{\mathrm{i}}-\mathrm{Ba} 1-\mathrm{Ba} 2^{\mathrm{vii}}$ & $36.31(2)$ & $\mathrm{I} 2^{\mathrm{ix}}-\mathrm{Ba} 2-\mathrm{Ba} 2^{\mathrm{vi}}$ & $108.287(17)$ \\
\hline 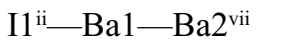 & $111.36(4)$ & $\mathrm{I} 2^{\mathrm{x}}-\mathrm{Ba} 2-\mathrm{Ba} 2^{\mathrm{vi}}$ & $108.049(12)$ \\
\hline 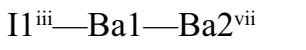 & $111.36(4)$ & $\mathrm{I} 2^{\mathrm{xi}}-\mathrm{Ba} 2-\mathrm{Ba} 2^{\mathrm{vi}}$ & $160.488(17)$ \\
\hline $\mathrm{I} 1^{\mathrm{iv}}-\mathrm{Ba} 1-\mathrm{Ba}^{\mathrm{vii}}$ & $171.58(2)$ & 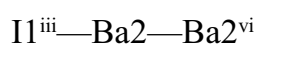 & $91.216(11)$ \\
\hline $\mathrm{I} 2^{\mathrm{v}}-\mathrm{Ba} 1-\mathrm{Ba} 2^{\mathrm{vii}}$ & $52.41(3)$ & $\mathrm{I} 1^{\mathrm{iv}}-\mathrm{Ba} 2-\mathrm{Ba} 2^{\mathrm{vi}}$ & $53.62(2)$ \\
\hline $\mathrm{I} 2^{\mathrm{vi}}-\mathrm{Ba} 1-\mathrm{Ba} 2^{\mathrm{vii}}$ & $106.13(2)$ & $\mathrm{I} 1^{\mathrm{v}}-\mathrm{Ba} 2-\mathrm{Ba} 2^{\mathrm{vi}}$ & $107.812(11)$ \\
\hline $\mathrm{I} 2-\mathrm{Ba} 1-\mathrm{Ba} 2^{\mathrm{vii}}$ & $106.13(2)$ & $\mathrm{I} 1-\mathrm{Ba} 2-\mathrm{Ba}^{\mathrm{vi}}$ & $55.009(12)$ \\
\hline $\mathrm{O}^{\mathrm{i}}-\mathrm{Ba} 1-\mathrm{Ba} 2^{\mathrm{i}}$ & $36.31(2)$ & $\mathrm{Ba} 1^{\mathrm{ix}}-\mathrm{Ba} 2-\mathrm{Ba}^{\mathrm{vi}}$ & $59.152(19)$ \\
\hline $\mathrm{I} 1^{\mathrm{ii}}-\mathrm{Ba} 1-\mathrm{Ba} 2^{\mathrm{i}}$ & $171.58(2)$ & $\mathrm{O}-\mathrm{Ba} 2-\mathrm{Ba} 2^{\mathrm{v}}$ & $34.36(12)$ \\
\hline $\mathrm{I} 1^{\mathrm{iii}}-\mathrm{Ba} 1-\mathrm{Ba}^{2}$ & $111.36(4)$ & $\mathrm{I} 2^{\mathrm{ix}}-\mathrm{Ba} 2-\mathrm{Ba} 2^{\mathrm{v}}$ & $108.287(17)$ \\
\hline $\mathrm{I} 1^{\mathrm{iv}}-\mathrm{Ba} 1-\mathrm{Ba} 2^{\mathrm{i}}$ & $111.36(4)$ & $\mathrm{I} 2^{\mathrm{x}}-\mathrm{Ba} 2-\mathrm{Ba} 2^{\mathrm{v}}$ & $160.488(17)$ \\
\hline $\mathrm{I} 2^{\mathrm{v}}-\mathrm{Ba} 1-\mathrm{Ba} 2^{\mathrm{i}}$ & $106.13(2)$ & $\mathrm{I} 2^{\mathrm{xi}}-\mathrm{Ba} 2-\mathrm{Ba} 2^{\mathrm{v}}$ & $108.049(12)$ \\
\hline $\mathrm{I} 2^{\mathrm{vi}}-\mathrm{Ba} 1-\mathrm{Ba} 2^{\mathrm{i}}$ & $106.13(2)$ & $\mathrm{I} 1^{\mathrm{iii}}-\mathrm{Ba} 2-\mathrm{Ba} 2^{\mathrm{v}}$ & $53.62(2)$ \\
\hline $\mathrm{I} 2-\mathrm{Ba} 1-\mathrm{Ba} 2^{\mathrm{i}}$ & $52.41(3)$ & $\mathrm{I} 1^{\mathrm{iv}}-\mathrm{Ba} 2-\mathrm{Ba} 2^{\mathrm{v}}$ & $91.216(11)$ \\
\hline $\mathrm{Ba} 2^{\mathrm{vii}}-\mathrm{Ba} 1-\mathrm{Ba} 2^{\mathrm{i}}$ & $61.70(4)$ & $\mathrm{I} 1^{\mathrm{v}}-\mathrm{Ba} 2-\mathrm{Ba}^{\mathrm{v}}$ & $55.009(12)$ \\
\hline 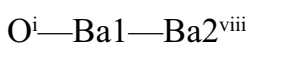 & $36.31(2)$ & $\mathrm{I} 1-\mathrm{Ba} 2-\mathrm{Ba}^{\mathrm{v}}$ & $107.812(11)$ \\
\hline $\mathrm{I} 1^{\mathrm{ii}}-\mathrm{Ba} 1-\mathrm{Ba} 2^{\text {viii }}$ & $111.36(4)$ & $\mathrm{Ba} 1^{\mathrm{ix}}-\mathrm{Ba} 2-\mathrm{Ba} 2^{\mathrm{v}}$ & $59.152(19)$ \\
\hline 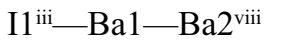 & $171.58(2)$ & $\mathrm{Ba} 2^{\mathrm{vi}}-\mathrm{Ba} 2-\mathrm{Ba}^{\mathrm{v}}$ & 60.0 \\
\hline $\mathrm{I} 1^{\mathrm{iv}}-\mathrm{Ba} 1-\mathrm{Ba} 2^{\text {viii }}$ & $111.36(4)$ & $\mathrm{Ba} 2^{\mathrm{x}}-\mathrm{I} 1-\mathrm{Ba}^{2 \mathrm{xi}}$ & $72.76(4)$ \\
\hline $\mathrm{I} 2^{\mathrm{v}}-\mathrm{Ba} 1-\mathrm{Ba} 2^{\mathrm{viii}}$ & $106.13(2)$ & $\mathrm{Ba} 2^{\mathrm{x}}-\mathrm{I} 1-\mathrm{Ba} 1^{\mathrm{xii}}$ & $104.53(4)$ \\
\hline $\mathrm{I} 2^{\mathrm{vi}}-\mathrm{Ba} 1-\mathrm{Ba} 2^{\mathrm{viii}}$ & $52.41(3)$ & 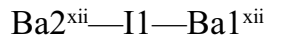 & $104.53(4)$ \\
\hline $\mathrm{I} 2-\mathrm{Ba} 1-\mathrm{Ba} 2^{\mathrm{viii}}$ & $106.13(2)$ & $\mathrm{Ba} 2^{\mathrm{x}}-\mathrm{I} 1-\mathrm{Ba}^{\mathrm{vi}}$ & $156.07(2)$ \\
\hline $\mathrm{Ba} 2^{\text {vii }}-\mathrm{Ba} 1-\mathrm{Ba} 2^{\text {viii }}$ & $61.70(4)$ & $\mathrm{Ba} 2^{\mathrm{xii}}-\mathrm{I} 1-\mathrm{Ba}^{\mathrm{vi}}$ & $103.52(3)$ \\
\hline $\mathrm{Ba} 2^{\mathrm{i}}-\mathrm{Ba} 1-\mathrm{Ba} 2^{\text {viii }}$ & $61.70(4)$ & $\mathrm{Ba} 1^{\mathrm{xii}}-\mathrm{I} 1-\mathrm{Ba}^{\mathrm{vi}}$ & $99.29(4)$ \\
\hline $\mathrm{O}-\mathrm{Ba} 2-\mathrm{I} 2^{\mathrm{ix}}$ & $93.6(3)$ & $\mathrm{Ba} 2^{\mathrm{x}}-\mathrm{I} 1-\mathrm{Ba} 2$ & $103.52(3)$ \\
\hline $\mathrm{O}-\mathrm{Ba} 2-\mathrm{I} 2^{\mathrm{x}}$ & $140.63(2)$ & $\mathrm{Ba} 2^{\mathrm{xii}}-\mathrm{I} 1-\mathrm{Ba} 2$ & $156.07(2)$ \\
\hline $\mathrm{I} 2^{\mathrm{ix}}-\mathrm{Ba} 2-\mathrm{I} 2^{\mathrm{x}}$ & $89.79(3)$ & $\mathrm{Ba} 1^{\mathrm{xii}}-\mathrm{I} 1-\mathrm{Ba} 2$ & $99.29(4)$ \\
\hline $\mathrm{O}-\mathrm{Ba} 2-\mathrm{I} 2^{\mathrm{xi}}$ & $140.63(2)$ & 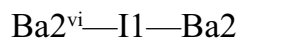 & $69.98(2)$ \\
\hline $\mathrm{I} 2^{\mathrm{ix}}-\mathrm{Ba} 2-\mathrm{I} 2^{\mathrm{xi}}$ & $89.79(3)$ & $\mathrm{Ba} 2^{\mathrm{i}}-\mathrm{I} 2-\mathrm{Ba} 2^{\mathrm{iii}}$ & $109.018(17)$ \\
\hline $\mathrm{I} 2^{\mathrm{x}}-\mathrm{Ba} 2-\mathrm{I} 2^{\mathrm{xi}}$ & $78.50(2)$ & $\mathrm{Ba} 2^{\mathrm{i}}-\mathrm{I} 2-\mathrm{Ba} 2^{\mathrm{xiii}}$ & $109.018(17)$ \\
\hline $\mathrm{O}-\mathrm{Ba} 2-\mathrm{I} 1^{\mathrm{iii}}$ & $84.4(2)$ & $\mathrm{Ba} 2^{\mathrm{iii}}-\mathrm{I} 2-\mathrm{Ba} 2^{\mathrm{xiii}}$ & $140.98(3)$ \\
\hline $\mathrm{I} 2^{\mathrm{ix}}-\mathrm{Ba} 2-\mathrm{I} 1^{\mathrm{iii}}$ & $141.96(2)$ & $\mathrm{Ba} 2 \mathrm{i}-\mathrm{I} 2-\mathrm{Ba} 1$ & $70.05(3)$ \\
\hline $\mathrm{I} 2^{\mathrm{x}}-\mathrm{Ba} 2-\mathrm{I} 1^{\mathrm{iii}}$ & $115.40(4)$ & 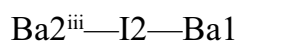 & $100.378(13)$ \\
\hline
\end{tabular}




$$
\begin{aligned}
& \mathrm{I} 2^{\mathrm{xi}}-\mathrm{Ba} 2-\mathrm{I} 1^{\mathrm{iii}} \\
& \mathrm{O}-\mathrm{Ba} 2-\mathrm{I} 1^{\mathrm{iv}} \\
& \mathrm{I} 2^{\mathrm{ix}}-\mathrm{Ba} 2-\mathrm{I} 1^{\mathrm{iv}} \\
& \mathrm{I} 2^{\mathrm{x}}-\mathrm{Ba} 2-\mathrm{I} 1^{\mathrm{iv}} \\
& \mathrm{I}^{\mathrm{xi}}-\mathrm{Ba} 2-\mathrm{I} 1^{\mathrm{iv}} \\
& \mathrm{I} 1^{\mathrm{iii}}-\mathrm{Ba} 2-\mathrm{I} 1^{\mathrm{iv}} \\
& \mathrm{O}-\mathrm{Ba} 2-\mathrm{I} 1^{\mathrm{v}}
\end{aligned}
$$

$69.600(17)$

$84.4(2)$

$141.96(2)$

$69.600(17)$

$\mathrm{Ba} 2^{\mathrm{xii}}-\mathrm{I} 2-\mathrm{Ba} 1$
$\mathrm{Ba} 1^{\mathrm{ix}}-\mathrm{O}-\mathrm{Ba}^{\mathrm{v}}$
$\mathrm{Ba} 1^{\mathrm{ix}}-\mathrm{O}-\mathrm{Ba} 2$
$\mathrm{Ba} 2^{\mathrm{v}}-\mathrm{O}-\mathrm{Ba} 2$
$\mathrm{Ba} 1^{\mathrm{ix}}-\mathrm{O}-\mathrm{Ba}^{\mathrm{vi}}$
$\mathrm{Ba} 2^{\mathrm{v}}-\mathrm{O}-\mathrm{Ba}^{\mathrm{vi}}$
$\mathrm{Ba} 2-\mathrm{O}-\mathrm{Ba}^{\mathrm{vi}}$

$100.378(13)$

$107.6(3)$

$107.6(3)$

$111.3(2)$

$107.6(3)$

$111.3(2)$

$111.3(2)$

Symmetry codes: (i) $x, y, z-1$; (ii) $y,-x+y+1, z-1 / 2$; (iii) $x-y, x, z-1 / 2$; (iv) $-x+1,-y+1, z-1 / 2$; (v) $-x+y,-x+1, z$; (vi) $-y+1, x-y+1, z$; (vii) $-x+y,-x+1$, $z-1$; (viii) $-y+1, x-y+1, z-1$; (ix) $x, y, z+1$; (x) $y,-x+y, z+1 / 2$; (xi) $x-y, x, z+1 / 2$; (xii) $-x+1,-y+1, z+1 / 2$; (xiii) $y,-x+y, z-1 / 2$. 